\title{
Eficiência e Gestão Pública em Saúde na APS
}

\author{
Allan Claudius Queiroz Barbosa, Aluísio Gomes da Silva Junior, Maria \\ Aparecida Turci, Philipe Scherrer Mendes
}

\section{RESUMO}

O cenário adverso da saúde no país, agravado pela fragilização do Sistema Único de Saúde (SUS), que, a despeito de tal contexto, tenta fazer frente à maior tragédia sanitária de nossa história recente, tem neste documento uma posição para reflexão da Rede de Pesquisa em Atenção Primária à Saúde (APS) da ABRASCO, acerca da Eficiência e Gestão Pública no contexto da APS. Estas notas abordam o contexto da APS e o agravamento com a pandemia da COVID-19, reforçando a importância do debate sobre subfinanciamento com recursos públicos e visa a potencializar os objetivos e valores sociais que perpassam a APS universal e de qualidade. Abordam também a compreensão do conceito de eficiência e sua adoção no contexto da APS, com foco na geração de serviços e cuidados com os recursos humanos, materiais, físicos e financeiros disponíveis às entregas e resultados decorrentes. Nessa perspectiva, será abordada a premissa de que mensurar a eficiência é uma maneira capaz de estimular resultados ótimos com os recursos disponíveis. No caso da APS, são mensuráveis os efeitos e impactos sobre os demais níveis de atenção. Ainda, a discussão sobre a Gestão Pública no contexto da APS é realizada considerando os diferentes formatos possíveis e seus efeitos à luz dos preceitos preconizados para uma saúde pública e universal. Por último, estas notas apresentam uma posição para o debate na Rede APS.

Palavras-chave: APS, Eficiência, Gestão Pública

\section{ABSTRACT}

The adverse health scenario in the country, aggravated by the weakening of the Unified Health System (SUS), which, despite such a context, tries to face the greatest health tragedy in our recent history, has in this document a position for reflection by the Research Network in Primary Health Care (PHC) of ABRASCO, about Efficiency and Public Management in the context of PHC. These notes address the context of PHC and the worsening with the COVID-19 pandemic, reinforcing the importance of the debate on underfunding with public resources and aiming to enhance the social goals and values that permeate universal and quality PHC. They also address the understanding of the concept of efficiency and its adoption in the context of PHC, focusing on the generation of services and care with human, material, physical and financial resources available for deliveries and resulting results. From this perspective, the premise that measuring efficiency is a way capable of stimulating optimal results with available resources will be addressed. In the case of PHC, the effects and impacts on other levels of care are measurable. Still, the discussion on Public Management in the context of PHC is carried out considering the different possible formats and their effects in light of the precepts advocated for universal and public health. Finally, these notes present a position for debate in the Rede APS.

Keywords: PHC, efficiency, public management.

Seção Debates e Discussões

Artigo convidado pela editoria científica
Revista da Rede APS 2021

Publicada em: 20/09/2021

DOI:10.14295/aps.v3i2.209

Allan Claudius Queiroz Barbosa (Universidade Federal de Minas Gerais, Belo Horizonte, MG, Brasil)

Aluísio Gomes da Silva Junior (Universidade Federal Fluminense, Niterói, RJ, Brasil)

Maria Aparecida Turci (UNIFENAS, Belo Horizonte, MG, Brasil)

Philipe Scherrer Mendes (Universidade Federal de Minas Gerais, Belo Horizonte, MG, Brasil)

Correspondência para:

Allan Claudius Queiroz Barbosa allan@ufmg.br 


\section{O Contexto da APS e o quadro}

\section{ATUAL DO SUS NO AMBIENTE DA PANDEMIA DA COVID-19}

O atual contexto da situação sanitária brasileira, além da grave crise sanitária que passou a dominar o debate a partir de março de 2020, coloca em discussão o papel da APS e do próprio SUS, ambos com seus princípios referendados pela Constituição Federal de 1988, que adotou a descentralização e consequentemente ampliou a cobertura dos serviços de saúde por meio de novas unidades básicas pelos municípios (MENDES, 2002).

Os princípios da universalidade de acesso, da integralidade da assistência e da equidade garantiram o acesso aos serviços públicos de saúde a qualquer cidadão, i.e., a um conjunto de ações e serviços preventivos e curativos, individuais e coletivos, sem preconceitos ou privilégios de qualquer espécie, por meio da municipalização da saúde, acesso esse que passou a ocorrer a partir do nível primário de atenção. Consequentemente, a criação do Programa Saúde da Família em 1994, posteriormente denominado Estratégia Saúde da Família, institucionalizou a política nacional de atenção primária à saúde.

Desde então, têm sido observados avanços tanto na garantia de atendimento pelo aumento da cobertura como nos parâmetros de melhoria

\footnotetext{
1 Marinho (2017) acrescenta que a proposta "planos de saúde acessíveis" se deu sob um enfoque econômico, cercada de incertezas sobre seu impacto na saúde da população. A análise da literatura evidencia a existência de riscos de degradação da saúde dos mais pobres e de piora nas condições de financiamento do Sistema Único de Saúde (SUS).

${ }^{2}$ O subfinanciamento é medido a partir do gasto público com saúde pública como percentual do produto interno bruto (PIB) e como per capita, ambos em comparação a países com sistemas públicos de saúde semelhantes. A este respeito, ver Marques (2017) e Barros e Piola (2016).
}

3

http://189.28.128.100/dab/docs/portaldab/doc da qualidade dos serviços prestados. Melo et al. (2019) indicam que, a partir de 2013, notadamente em razão da crise econômica e institucional, as medidas restritivas adotadas em anos subsequentes por meio do congelamento de gastos com saúde via emenda constitucional (2016); mudança nos blocos de financiamento do SUS em 2017; crescimento das chamadas clínicas populares privadas e proposições de desregulação da saúde suplementar que permitem a adoção de planos populares1; acrescidas das alterações na Política Nacional de Atenção Básica (PNAB) em 2017, exerceram impacto direto na oferta de serviços. Isso, em um ambiente de subfinanciamento da saúde tido como crônico. 2

Mais recentemente, em 2019, a criação do programa "Previne Brasil" propôs um novo modelo de financiamento alterando o repasse das transferências para os municípios, que passaram a ser distribuídas com base em três critérios: captação ponderada, pagamento por desempenho e incentivo para ações estratégicas3. Uma das justificativas dessa proposta foi exatamente a possibilidade de fomentar a avaliação da eficiência dos sistemas de saúde locais, embora tenha utilizado uma abordagem financeira de eficiência4 (Harzheim et al., 2020).

Nesse contexto de grandes transformações do modus operandi da APS, a chegada, em fins de

umentos/financiamento/portarias/prt_2979_1 2_11_2019.pdf

4 A proposta de financiamento concretizada através do Previne contou com a colaboração de profissionais do Banco Mundial e teve como um dos seus pilares os achados de estudo desta instituição, encontrado em: Banco Mundial. Um ajuste justo: Análise da eficiência e equidade do gasto público no Brasil [página na Internet]. 2017 Disponível em: https://www.worldbankorg/pt/country/brazil/ publication/brazil-expenditure-review-report 
de 2019, de um novo vírus da família coronavírus - SARS-CoV-2, levou a Organização Mundial de Saúde (OMS) a declarar pandemia em 11 de março de 2020 (LIMA, 2020; BELASCO e FONSECA, 2020).

Esperava-se que a APS tivesse papel fundamental nesse cenário tanto por suas características como pela sua importância no desenvolvimento das atividades de vigilância, prevenção e promoção da saúde. Ou seja, o modelo preconizado de atuação com base em equipes multiprofissionais permitiria fortalecer o papel da APS no enfrentamento e combate da pandemia pela COVID-19, destacando seu caráter comunitário e de proximidade. Entretanto, a ausência de uma coordenação nacional e a excessiva fragmentação de ações e medidas de âmbito local, associadas ao agravamento da crise econômica, exerceram impacto sobre os indicadores de saúde, de desigualdade social, renda do trabalho, concentração da renda e taxas de desemprego 5.

À luz desse quadro, é de fundamental importância requalificar o debate em torno da APS e de seu papel primordial como modelo de atenção. Além das ações e serviços desenvolvidos por profissionais capacitados e comprometidos, por exemplo, torna-se essencial observar aspectos ligados à avaliação da eficiência dos serviços prestados como forma de potencializar o uso considerado adequado de recursos públicos.

Ou seja, é relevante entender o conceito de eficiência e as formas pelas quais é possível mensurá-la, principalmente considerando o contexto da saúde pública. A eficiência analisa o que foi produzido, dados os recursos disponíveis, com o que poderia ter sido produzido com os mesmos recursos, isto é, a eficiência é uma medida relativa que tem

5 a esse respeito, ver, por exemplo: https://portal.fgv.br/artigos/qual-foi-efeitopandemia-sobre-mercadotrabalho?utm_source=portalfgv\&utm_medium=fgvnoticias\&utm_campaign =fgvnoticias-2020-10-14 relação com certas comparações de produtividade (PEÑA, 2008). Em um ambiente de escassez de recursos e mesmo de crise econômica, medir a eficiência é uma forma eficaz para estimular resultados ótimos com recursos disponíveis.

\section{O CONCEITO DE EFICIÊNCIA, SUA}

\section{POSSÍVEL MENSURAÇÃO E ADOÇÃO NO CONTEXTO DA APS}

O debate sobre eficiência tem sido pautado fundamentalmente na lógica de recursos vis à vis os diferentes níveis de atenção, considerando a dicotomia público-privado que norteia a organização do sistema de saúde. Isso, dentro da clássica concepção de demanda infinita que perpassa a oferta dos serviços de saúde. Ora, nesse ambiente, buscar padrões de eficiência na alocação e uso de recursos com qualidade e satisfação de usuários torna-se uma alternativa e não um fim em si mesmo, isto é, ser eficiente não substitui as demandas e necessidades intrínsecas ao campo da saúde, notadamente à atenção primária à saúde. ${ }^{6}$

Tasca et al. (2020) mostraram que em 2018 o Brasil alcançou mais de $60 \%$ da população brasileira com a Estratégia da Saúde da Família. Dado o significativo esforço financeiro envolvido nessa marca, discutir estratégias para a ampliação e melhoria dos serviços prestados deve necessariamente incorporar a busca pela eficiência.

De forma geral, Rodrigues e Barbosa (2021, p. 219) apontam que a eficiência "está pautada na oferta dita 'ótima' de bens e serviços de saúde para o consumidor ou usuário dos serviços ao menor custo possível (eficiência técnica e alocativa) em um mercado (saúde)

6 Neste aspecto, é importante diferenciar eficiência e desempenho, sendo este conceito associado à estratégia e o que é esperado em termos de resultados organizacionais, conforme observou Rodrigues (2016). 
caracterizado por economias de escala e escopo com barreiras de entrada."

Os autores indicam ainda que uma abordagem de cariz econômico é usual, por meio do uso de funções paramétricas que determinem a eficiência econômica absoluta. Ainda, o uso de programas matemáticos não paramétricos com uma avaliação comparativa tem sido bastante difundido ${ }^{7}$, dada a dificuldade de modelos paramétricos em obter grandes amostras com maior especificação das funções que avaliem eficiência e ineficiência. (RODRIGUES e BARBOSA, 2021).

Barbosa et al. (2020) observaram a importância de entender o conceito de eficiência e as formas pelas quais é possível mensurá-la. A eficiência é uma medida relativa que tem relação com certas comparações de produtividade (PEÑA, 2008). Ou seja, mensurar a eficiência é uma forma eficaz para estimular resultados ótimos com os recursos disponíveis. No caso da APS, são visíveis os efeitos e impactos sobre os demais níveis de atenção tais como, por exemplo, a redução de internações e a minimização do uso de soluções de alto custo financeiro.

Entretanto, é usual a utilização de métricas que não necessariamente observam as especificidades da APS em termos de seus atributos e dinâmica de prestação de serviços ao usuário. Ao se discutir eficiência em saúde, especificamente em seu nível mais básico e amplo, é fundamental levar em consideração a lógica envolvida no serviço prestado e a que fim ele se destina.

Um primeiro ponto, destacado por David (2015) e que merece ser abordado, é a característica incerta da saúde. Existe uma incerteza

\footnotetext{
7 Tem sido usual estudos para mensurar eficiência que se utilizam de modelos matemáticos e o Data Envelopment Analysis (DEA) se destaca dentre eles. Neste caso, Rodrigues e Barbosa (2021) apontam que o DEA evidencia que eficiência se dá através da relação ótima entre as variáveis examinando inputs, entendidos como recursos, e outputs, que seriam os produtos. Tem-se como princípio a ideia de relativa homogeneidade quanto às
}

relacionada à ocorrência da doença, outra envolvida no diagnóstico e uma outra diz respeito ao tratamento. Isso, inevitavelmente, é determinante do resultado almejado, que é a saúde. Esse é outro ponto relevante: as discussões relacionadas à identificação de resultados devem ter em mente que o desejável não é o cuidado tomado, mas a saúde da população.

Nesse sentido, a complexidade intrínseca à análise e ao tema demanda esforço que vai além da identificação de um bom método, pois passa pelo entendimento da sua lógica, seu desenho de funcionamento e a resolutividade que se obtém.

Silva et al (2018), por exemplo, abordam os fatores que influenciam a eficiência na aplicação de recursos na APS segundo o uso da metodologia DEA, que utiliza as variáveis populacionais dos municípios, número de médicos por estabelecimento e consultas por estabelecimento, além de indicador de desenvolvimento municipal de saúde. Ou seja, de forma geral, a abordagem da seleção de variáveis segue usualmente a opinião de especialistas ou até mesmo da disponibilidade de dados para modelar as análises.

Assim, é primordial ir além da modelagem clássica e incorporar múltiplos indicadores de insumo e de produto, dada a possibilidade de utilizar seus atributos preconizados por Starfield (2002). Com efeito, a escolha das variáveis de entrada, inputs, e saída, outputs, deve mostrar apontar o que é relevante para o desempenho da APS, considerando que a eficiência só é, de fato, efetiva quando ligada aos fatores capazes de gerar desempenho (BARBOSA et al, 2020).

tarefas realizadas, objetivos, condições de mercado e autonomia na tomada de decisões, mas são diferentes nas quantidades dos recursos consumidos (inputs) e das saídas produzidas (outputs), em função de aspectos internos destes hospitais (COOK; SEIFORD, 2009). 
Por isso, a análise prévia de variáveis deve ser feita segundo os olhares de decisores, especialistas e analistas, sempre voltados ao atendimento das necessidades do usuário. 0 decisor deve verificar se não foi omitida nenhuma variável considerada imprescindível. Nessa perspectiva, discutir eficiência vai além da dimensão econômica, devendo ser realmente relevante para se determinar o desempenho da APS. Ou seja, é primordial uma escolha de variáveis que leve em conta os diferentes e possíveis olhares que abarcam a APS, sob diferentes pontos de vista. Conforme dizem Barbosa et al (2020, p. 3),

"A análise prévia de possíveis conjuntos de variáveis é uma etapa fundamental, a ser feita conjuntamente por decisores, especialistas e analistas. Em qualquer método o decisor deve ser confrontado com o resultado e verificar a sua coerência. Deve verificar se não foi omitida nenhuma variável que considere imprescindível, se há relação causal entre todos os pares input-output selecionados."

O debate sobre Atenção Primária à Saúde no Brasil tem a seu dispor, a partir das bases de dados nacionais e da utilização crescente de instrumentos de avaliação, uma série de indicadores que se apresentam como alternativas de variáveis para a avaliação de efetividade. O Quadro 1 sistematiza alguns exemplos:

Quadro 1 - Exemplos de inputs e outputs para avaliações de eficiência na APS

\begin{tabular}{|c|c|c|}
\hline Inputs (insumos) & Outputs (saídas) & Resultados \\
\hline $\begin{array}{l}\text { número de médicos, } \\
\text { enfermeiros, equipe } \\
\text { multiprofissional e ACS; } \\
\text { número de número de } \\
\text { equipes de atenção } \\
\text { primária } \\
\text { - } \text { número de Unidades } \\
\text { Básicas de Saúde; } \\
\text { disponibilidade de } \\
\text { materiais, equipamentos } \\
\text { e medicamentos; } \\
\text { gastos municipais com } \\
\text { - } \quad \text { regS; } \\
\text { - características da } \\
\text { população atendida } \\
\text { (composição etária, } \\
\text { cobertura por } \\
\text { saneamento básico, } \\
\text { vulnerabilidade social) }\end{array}$ & $\begin{array}{l}\text { número de famílias } \\
\text { acompanhadas; } \\
\text { número de visitas } \\
\text { domiciliares; } \\
\text { cobertura de } \\
\text { consultas; } \\
\text { - solicitação de exames } \\
\text { a grupos específicos; } \\
\text { qualidade do } \\
\text { atendimento } \\
\text { (proporção de } \\
\text { consultas com exames } \\
\text { dos pés em diabéticos, } \\
\text { proporção de } \\
\text { consultas com exames } \\
\text { das mamas); } \\
\text { cobertura de pré-natal } \\
\text { (7 ou mais consultas); } \\
\text { cobertura vacinal. }\end{array}$ & 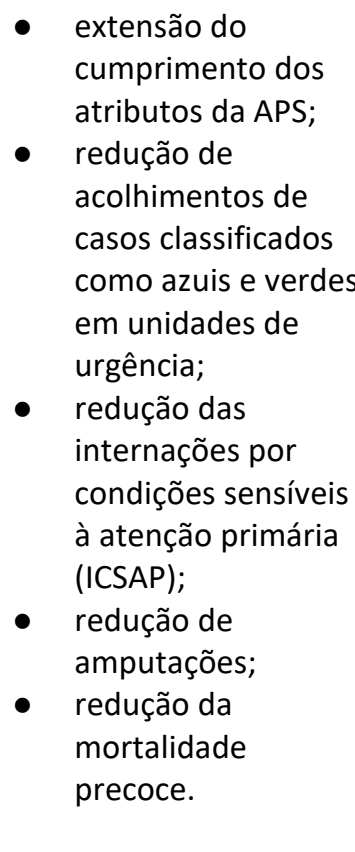 \\
\hline
\end{tabular}

Fonte: Elaborado pelos autores a partir de Braga et al. (2015) e Cabral et al. (2019)

Nessa perspectiva, pensar na lógica da APS pressupõe que a avaliação de eficiência deve ser observada a partir de fatores considerados na perspectiva da sua dinâmica, considerando somente aquilo que é realmente relevante para determinar o desempenho esperado. Fatores tais como porte populacional, grau de desenvolvimento municipal, vulnerabilidade da 
população, composição etária da população e distribuição dos profissionais de saúde podem afetar a eficiência (DIAS, 2010).

Vale destacar a heterogeneidade na qualidade da atenção primária ao longo de todo o território nacional, fator que deve ser considerado e tratado na análise de eficiência. Isso, porque é reconhecida a discrepância entre os 5570 municípios brasileiros, que possuem problemas que vão da escassez de profissionais a restrições orçamentárias (TASCA et al. (2020). Há que se considerar, também, o contexto com diferentes formas de organização institucional e normativa da prestação de serviços de saúde na APS.

Miclos et al. (2015) destacam a importância em se avançar nos estudos que avaliam o desempenho da Atenção Primária em Saúde com o uso do instrumental DEA. As autoras ressaltam as dificuldades nas escolhas dos melhores inputs e outputs, de forma a permitir uma boa análise da eficiência na APS. Destacam também que pouco se produziu até então, com significativa heterogeneidade nas definições de insumo, produto e unidade de análise (DMUs), o que dificulta a comparabilidade dos resultados obtidos pelos diferentes trabalhos. Nesse sentido, fica o convite a se avançar nesse aspecto da agenda. Por fim, é importante também refletir que a oferta de serviços mais eficientes não significa que estes apresentem bons parâmetros de qualidade e tampouco induzam à equidade (DIAS, 2010).

\section{Gestão Pública no CONTEXto dA}

APS

A "Contribuição da Rede de Pesquisa em APS da ABRASCO para a formulação de uma agenda política estratégica para a APS no SUS", de 2018, mostrou, utilizando-se de dados da avaliação externa do Programa Nacional de Melhoria do Acesso e da Qualidade da Atenção Básica (PMAQ), em seus resultados do primeiro e segundo ciclos da avaliação externa, a tendência ao uso da administração indireta na gestão da APS por municípios, visando primordialmente a não atingir os limites da Lei de Responsabilidade Fiscal e da administração pública tradicional. Os dados do documento da Rede APS apontavam

Em 2013 (BRASIL, 2014), 1.216 municípios brasileiros $(21,8 \%)$ tinham estabelecido contratos de gestão com organizações sociais (OS) na área de saúde. Outro dado que chama atenção é que entre os 39 municípios com mais de 500.000 habitantes, 22 deles $(56,4 \%)$ contrataram serviços de saúde por meio de OS (BRASIL, 2014). Na APS, as organizações sociais e fundações estatais de direito privado (FEDP) assumiram a gestão operacional no Estado de São Paulo, no Município do Rio de Janeiro e no Estado da Bahia. (p. 416)

Naquele momento, havia controvérsia acerca dos formatos institucionais, sem consenso sobre seus efeitos na prestação de serviços de saúde. A ausência de um marco legal voltado à formalização dos papéis e atribuições dos atores e de um marco lógico de avaliação baseado na eficiência ainda colocam dúvidas sobre os efeitos destes modelos.

Esse debate sobre eventuais modelos alternativos à gestão direta propostos a partir da segunda metade da década de 1990 no bojo do amplo debate internacional sobre o papel do estado teve, no ideário da administração pública gerencial (BRESSER, 1996), sua referência no Brasil. Pautado fundamentalmente pela crise fiscal do Estado (HOOD, 1995; POLLITT e BOUCKAERT, 2002, dentre outros), trouxe à tona ainda a disputa de investidores privados por parcelas de serviços e bens públicos.

Associados ao processo de descentralização da saúde e seus efeitos no processo de trabalho na saúde universal, integral e participativa, os desdobramentos na relação público-privado diante desses novos formatos gerenciais (TEIXEIRA et al, 2018) trouxeram impactos e grande discussão sobre sua viabilidade.

Seja pela forma de administração pública direta, seja pela via indireta - por meio de autarquias, fundações públicas, consórcios Públicos, sociedades de economia mista, agências 
executivas, agências reguladora, empresas Públicas e contrato de gestão - ou mesmo pela via privada - por meio de fundações privadas, terceirização e parcerias, organizações sociais, organização da sociedade civil de interesse público, serviços ou contrato privados, dentre outras modalidades - é imprescindível assegurar os mecanismos e condições de uma oferta de serviços de qualidade e que atendam ao que é preconizado pela APS, em padrões de eficiência compatíveis às melhores práticas de gestão de recursos públicos. Mesmo que a própria estrutura do SUS considere simultaneamente uma lógica (público-privado) em simultâneo.

Dessa forma, estudar a eficiência dos diferentes formatos de gestão auxilia o debate qualificado e permite avançar em direção ao que se espera em termos de resultados de saúde. Ramos e De Seta (2019), por exemplo, apresentaram estudo comparativo de desempenho com diversos indicadores entre capitais que adotaram a flexibilização da gestão da Atenção Primária por Organizações Sociais (OS) no Rio de Janeiro (RJ) e São Paulo (SP), e aquelas que mantiveram a Administração Direta (AD): Belo Horizonte (MG) e Vitória (ES). Neste estudo, os autores constataram o melhor desempenho da Administração Direta.

Nessa perspectiva, e dentro do que a Rede APS já registrava em 2018, este debate sugere que é preciso abordar seus efeitos em um contexto de participação popular e fortalecimento dos mecanismos de regulação, independente do formato heterogêneo adotado pela gestão municipal na relação público-privada e na gestão de contratos.

Somem-se a isso os limites impostos aos gastos com pessoal nas três esferas de governo por meio de legislação específica, dificultando tanto a contratação de profissionais para expansão das ações e dos serviços de saúde como a

8 TRIBUNAL DE CONTAS DA UNIÃO. Relatório de Auditoria Operacional, TC 018. 739/2012-1. Brasília, 2012. Disponível em: http://www.tcu.gov.br/consultas/juris/doc remuneração em níveis compatíveis com o mercado (BARBOSA, 2015).

Para driblar esses limites, as gestões municipais têm lançado mão da terceirização das atividades de atenção primária à saúde. Relatório de Auditoria Operacional do Tribunal de Contas da União ${ }^{8}$ observou o crescimento das Organizações Sociais e da terceirização em atividades de saúde e suas conclusões mostram, dentre outros aspectos, ausência de controle e avaliação dos contratos e dos serviços e ausência de controle financeiro no repasse de recursos. Ou seja, é imperativo refletir sobre sua viabilidade, na medida em que as ferramentas e instrumentos que asseguram sua qualidade e efetividade não são devidamente adotadas pelos gestores públicos, e, por vezes, quando adotados, não conferem melhor eficiência, expondo os gestores a fraudes e quebras financeiras.

De forma geral, permanece como premissa inabalável a garantia da autonomia

da gestão municipal de saúde, notadamente na regulação da relação público-privada na gestão de contratos. Garantir a autonomia do gestor 'na ponta', assegurando indução naquilo que foge de seu escopo, deve ser feito observando práticas de governança com efetivo controle social e transparência dos procedimentos usuais de gestão. (REDE APS, 2018, pág. 417).

\section{UMA POSIÇÃO PARA O DEBATE NA REDE APS}

Agora, mais do que nunca, torna-se primordial garantir que os princípios preconizados historicamente na Declaração de Alma Ata sejam assegurados à luz do contexto global e das dificuldades sanitárias impostas pela pandemia

s/judoc/acord/20131129/ac_3239_47_13_p. doc. 
da COVID-19. Em nossa contribuição para a formulação de uma agenda política estratégica para a APS no SUS em 2018, já chamávamos a atenção para o acirramento do debate internacional acerca da universalização da saúde com dois polos distintos: sistema universal versus cobertura universal (GIOVANELLA e MACHADO, 2018).

Ou seja, uma APS que orienta a rede pressupõe assegurar uma abordagem integral dos sistemas universais, com a primazia pública no financiamento e prestação de serviços, sempre alinhada ao bem-estar social e econômico da sociedade. Isso, assegurado com uma gestão pública transparente, democrática, participativa e eficiente, visando a:

1. Garantir a presença da provisão pública na prestação dos serviços de saúde na rede, em especial na APS, em equilíbrio com os aparelhos e modelos de provisão não públicos, minimizando os efeitos da popularização de planos de saúde que não asseguram a continuidade do cuidado e dos processos de terceirização.

2. Assegurar, de forma normativa e operacional, os mecanismos de regulação da oferta de serviços não públicos, notadamente nos contratos e modelos de gestão, com o objetivo de garantir a assistência integral à população e evitar fraudes.

3. Fortalecer o papel dos estados em âmbito regional por meio do financiamento e da regulação da rede de serviços, proporcionando a integração da atenção primária aos demais níveis de atenção.

4. Fortalecer e implementar de forma efetiva o uso de ferramentas voltadas à eficiência na alocação de recursos na APS, apoiando iniciativas que estabeleçam 0 entendimento das especificidades dos serviços vis à vis os princípios norteadores da APS, tendo como pressupostos que os desfechos devem ter relevância na saúde e na qualidade de vida das pessoas, além de consonância com as necessidades dos usuários.

5. Os custos operacionais da APS são baixos quando comparados aos serviços de média e alta complexidades, embora os resultados econômicos sejam importantes em termos de redução de internações e mortes prematuras. Dessa forma, está justificada a alocação constante e em suficiência nesse nível de atenção.

6. Fomentar o desenvolvimento sistemático de estudos e pesquisas em diferentes perspectivas metodológicas que possam produzir indicadores comparativos para avaliar a eficiência dos modelos de gestão da APS. 


\section{REFERÊNCIAS}

Alfradique ME, Bonolo PF, Dourado I, Costa-Lima MF, Macinko J, Mendonça CS, Oliveira VB, Sampaio LFR, Simoni C, Turci MA. Internações por condições sensíveis à atenção primária: a construção da lista brasileira como ferramenta para medir o desempenho do sistema de saúde (Projeto ICSAP Brasil). Cad Saude Publica 2009; 25(6):1337-1349.

Barbosa, A. C. Q.; Kemper, E.; Mendes, P. S.; Rehem, R.; Tasca, R. Eficiência na produção de serviços em saúde e a escolha do método de avaliação - Um estudo em hospitais materno-infantis brasileiros. Brasília: OPAS, 2020 (texto para discussão).

Barbosa, A. C. Q. Recursos Humanos e Vínculos Profissionais Análise Descritiva em Hospitais de Pequeno Porte do Brasil. Belo Horizonte: UFMG, 2015 (tese de professor titular)

Barros, M. E. D.; Piola, S. F.. O financiamento dos serviços de saúde no Brasil. In: Marques, R. M.; Piola, S. F.; Carrilo Roa, A. (Orgs.). Sistemas de saúde no Brasil: organização e financiamento. Brasília: Abres; MS; Opas/OMS, 2016.

Belasco, G.S.; Fonseca, C.D. Coronavírus 2020. Rev. Bras. Enferm., Brasília , v. 73, n. 2, e2020n2, 2020. http://dx.doi.org/10.1590/0034-7167-2020730201.

Braga GB, Ferreira MAM, Braga BB. A Eficiência da Atenção Primária à Saúde: Avaliando Discrepâncias [Internet]. Vol. 7, Administração Pública e Gestão Social. 2015. Disponível em: http://dx.doi.org/10.21118/apgs.v7i2.776

Brasil. Ministério do Planejamento, Desenvolvimento e Gestão. Pesquisa de Informações Básicas Municipais - 2013. Rio de Janeiro: Ministério do Planejamento, Desenvolvimento e Gestão; 2014.

Cabral KFD, Ferreira MAM, Batista RS, Cerqueira FR. Atenção primária à saúde: uma análise a luz da eficiência técnica dos recursos no Estado de Minas Gerais. Revista de Gestão em Sistemas de Saúde [Internet]. 2019;8(2). Disponível em: https://periodicos.uninove.br/revistargss/article/view/14923

Cook W. D.; Seiford L. M. Data Envelopment Analysis (DEA) - Thirty Years. European Journal of Operational Research, v. 192, n. 1, pp. 1-17, 2009

David, G. C. Atenção primária nos municípios brasileiros entre 2007-2010: desempenho, gasto, eficiência e disparidades. Dissertação de Mestrado. Programa de Pós-Graduação em Saúde Coletiva. Brasília: Universidade de Brasília, 2015.

Dias RH. Eficiência da atenção primária à saúde nos municípios brasileiros. 2010 Apr 30 [cited 2021 Jul 27]. Disponível em: http://repositorio.unb.br/handle/10482/8364

Giovanella, L., Machado, C. V. Sistema universal de saúde e cobertura universal: desvendando pressupostos e estratégias. Ciência e Saúde Coletiva. 23 (6), junho 2018. https://doi.org/10.1590/141381232018236.05562018

Harzheim, Erno et al. Novo financiamento para uma nova Atenção Primária à Saúde no Brasil. Ciência \& Saúde Coletiva [online]. v. 25, n. 4, pp. 1361-1374. Acesso em 28 de julho de 2021.

Hood, Christopher. The "new public management" in the 1980s: variations on a theme. Accounting, Organizations and Society, v. 20, n. 2/3, p. 93-109, 1995.

Lima, C.A.O. Informações sobre o novo coronavírus (COVID-19). Radiol Bras, São Paulo, v. 53, n. 2, p. V-VI, Apr. 2020. https://doi.org/10.1590/0100-3984.2020.53.2e1 
Marinho, A. a crise do mercado de planos de saúde: devemos apostar nos planos populares ou no SUS?. Planejamento e Políticas Públicas, [S. I.], n. 49, 2017. Disponível em: //www.ipea.gov.br/ppp/index.php/PPP/article/view/953. Acesso em: 31 ago. 2021.

Marques, R. M. Notas exploratórias sobre as razões do subfinanciamento estrutural do SUS. planejamento e políticas públicas. n. 49, jul./dez. 2017

Melo, E. A.; Mendonça, M.H.M.; Teixeira, M. A crise econômica e a atenção primária à saúde no SUS da cidade do Rio de Janeiro, Brasil. Ciência \& Saúde Coletiva, 24(12):4593-4598, 2019

Mendes, E. V. A atenção primária à saúde no SUS. Fortaleza: Escola de Saúde Pública do Ceará, 2002.

Miclos P. V.; Calvo M.C.M.; Colussi C.F. Avaliação do desempenho da atenção primária em saúde através da análise envoltória de dados. Revista Eletrônica Gestão \& Saúde. 6(2):1749-63. 2015.

Peña, C. R. Um modelo de avaliação da eficiência da administração pública através do Método Análise Envoltória de Dados (DEA). Revista de Administração Contemporânea, Curitiba, v.12, n.1, p.83-106, 2008.

Pereira, L. C. B. Da administração pública burocrática à gerencial. Revista do Serviço Público, 47(1) janeiroabril de 1996.

Pollitt, C.; Bouckaert, G. Avaliando reformas da gestão pública: uma perspectiva internacional. Revista do Serviço Público, v. 53, n. 3, p. 5-30, 2002a.

Ramos, A. L. P., De Seta, M. H. Atenção primária à saúde e Organizações Sociais nas capitais da Região Sudeste do Brasil: 2009 e 2014. Cadernos de Saúde Pública [online]. 2019, v. 35, n. 4 [Acessado 25 Julho 2021] , e00089118. Disponível em: <https://doi.org/10.1590/0102-311X00089118>. Epub 08 Abr 2019. ISSN 1678-4464. https://doi.org/10.1590/0102-311X00089118.

Rede de Pesquisa em Atenção Primária à Saúde da Abrasco. Contribuição para uma agenda política estratégica para a Atenção Primária à Saúde no SUS. Saúde debate 42(spe1), set 2018. https://doi.org/10.1590/0103-11042018S128

Rodrigues, J. M., Barbosa, A. C Q. Recursos humanos e eficiência: um estudo em hospitais brasileiros de pequeno porte. Nova Economia, v.31, n.1, p. 217-245, 2021. Disponível em: https://revistas.face.ufmg.br/index.php/novaeconomia/article/view/6080. Acesso em: 20 set. 2021.

Rodrigues, J. M. Gestão de recursos humanos e desempenho hospitalar na perspectiva da eficiência: um estudo em hospitais brasileiros de pequeno porte. Belo Horizonte: UFMG, 2016 (tese de doutorado)

Silva, C. R.; Souza, T. C.; Lima, C. M. B. L; Filho, L. B. S. Fatores associados à eficiência na Atenção Básica em saúde, nos municípios brasileiros. Saúde debate 42(117) apr-Jun 2018.

Starfield B. Atenção primária: equilíbrio entre necessidades de saúde, serviços e tecnologia. Brasília: UNESCO, Ministério da Saúde; 2002.

Tasca, R.; Massuda, A.; Carvalho, W. M.; Buchweitz, C.; Harzheim, E. Recomendações para o fortalecimento da atenção primária à saúde no Brasil. Revista Panamericana Salud Publica, 44:1-8, 2020.

Teixeira, M.; Matta, G. C.; Silva-Junior, A. G. Modelos de gestão na Atenção Primária à Saúde: uma análise crítica sobre gestão do trabalho e produção em saúde In Mendonça, Maria Helena Magalhães de; Matta, Gustavo Corrêa; Gondim, Roberta; Giovanella, Ligia. Atenção primária à saúde no Brasil: conceitos, práticas e pesquisa. Rio de Janeiro: Fiocruz, 2018. p.117-142.

Tribunal de Contas da União. Relatório de Auditoria Operacional, TC 018. 739/2012-1. Brasília, 2012. Disponível em: http://www.tcu.gov.br/consultas/juris/doc s/judoc/acord/20131129/ac_3239_47_13_p. doc. 